rhagia of the eye, the anus, various folds of the skin, the nose, the ear, or mouth.

1. External Gonorrhœa. - External gonorrhœachaudepisse bâtarde - balanitis - posthite - balanoposthite.

The causes which produce more particularly this form are phymosis, congenital or acquired, the accumulation of natural secretions, the habit of masturbation.

Premonitory Signs. - A sensation of weight or uneasiness, an unusual degree of heat about the part, a distressing sense of itching, are the only signs referable to the early stage of the disease.

Symptoms.-Inflammation may extend to the whole lining of the prepuce, or be limited to a small portion of mucous surface. The membrane soon becomes red from injection, its natural secretion is exchanged for a discharge thicker and more creamy than that of uretritis, and incarceration within the prepuce, together with incipient decomposition, communicate to this liquid a particularly offensive and irritating character. Contact is painful, desquamation of the epithelium, rendering the organ exquisitely sensitive from the denudation of a number of nervous papillæ.

Complications.-It is not uncommon to find œdema of the lax subcutaneous cellular structure complicating the disease. Erysipelas is occasionally met with, doubling the size of the penis, and not unfrequently acquiring so much violence as to threaten or even produce gangrene.

But in contradistinction to urethral blenorrhagia, epididymitis is never the consequence of external gonorrhœa, and very seldom bubo.

Eczema, on the other hand, and herpes break out on the glans, stimulated into existence by the presence of the inflammation.

Condylomata and excresences of various kinds are the result, and may become the cause of balano. posthitis.

Chancre and mucous tubercle may also complicate or produce external gonorrhœa. It is not a little remarkable that the first of these affections gives rise in its vicinity to a perfectly simple inflammation, the secretions of which may be inoculated with impunity, whereas pus from the neighbouring chancre will constantly and at the same time give a specific sore by inoculation.

Differential Diagnosis. - From eczema or herpes præputialis, external gonorrhœa will be easily distinguished by the diffused character of the inflammation, the absence of vesicles of any kind during its first period, or of those small circular erosions which follow herpes, and which have more analogy of appearance with superficial chancre than with simple inflammation of the prepuce or glans.

The scalding in passing water concentrated in the fossa navicularis, a view of the discharge coming from the urethra in cases where the foreskin can be slightly drawn back, also the particular pain caused by the distension of urethra during erection, will suffice to establish the diagnosis between blenorrhagia and balano-posthitis, in which erections are free from uneasiness; pressure determines pain around the base of the glans, and the discharge comes from the cul de sac formed by the reflection of the mucous membrane.

As to chancre, it will be sometimes possible to obtain a rational diagnosis of its existence under an irreducible phymosis, from a certain degree of painful hardness in one particular spot of the corona glandis, from the presence of enlarged ganglions in the groins, rather uncommon in simple balinitis; but one sign only can be implicitly relied upon to distinguish absolutely simple external gonorrhœa from phymosis complicated with chancre, and that sigu is inoculation with the lancet.

\section{ON NEURALGIC AFFECTIONS OF THE URETHRA.}

By M. Civinle, Paris.

There are certain affections of the genito-urinary organs which, though of frequent occurrence, have not much attracted the attention of practical surgeons. This arises, in great measure, from their irregularity of progress, the variety of symptoms which they occasion, and the great difficulty often experienced in distinguishing them from other morbid conditions which give rise to analagous symptoms.

It is seldom possible to ascertain the precise cause of the affections now alluded to, which I shall call neuralgic, without, however, attaching much importance to the name. They generally occur in persons whose nervous system has been shaken by excessive venereal indulgence, or severe moral shocks, and more especially in those who have been previously subject to neuralgia in other parts of the body. Longcontinued irritation of the urethra and neck of the bladder, stricture in its incipient stage, habitual constipation, and diseases of the womb or rectum, are predisposing causes; but, on the other hand, they attack persons in whom we cannot discover any of the causes just mentioned.

The symptoms of urethral neuralgia are in general so vague and variable, that we are often obliged to base our diagnosis on indirect reasoning. In fact, the sensations experienced by the patient, and the urinary derangement under which he suffers, may equally depend on calculus in the bladder, some organic disease of the prostate, neck, or body of the bladder, partial paralysis of that organ, stricture, \&c. Still the peculiar tendency of the symptoms to shift may throw some light on the matter, and the surgeon must be guided by the irregular or even intermittent character of these symptoms, which appear and disappear, increase or diminish, without any evident cause, and much more quickly than in any other disease of the urinary apparatus. This is so true that the attention of the patient is often not directed to them until after the third or fourth attack. In an early stage he enjoys long intervals of health, the frequent desire to make water, with pain and diffculty in passing it, occurring only at intervals. Soon after these symptoms the patient experiences a distressing sensation of itching along the canal, with burning; this is chiefly felt near the glans, and may extend towards the pubes, groins, sacrum, or up to the kidneys. The urine is in general normal; these symptoms seldom last long enough to produce any effect on the general health; they disappear at last, and the patient gets well.

It is impossible to give any accurate account of the 
frequency, duration, or order of the attacks now the neuralgia; but to this point I shall revert herealluded to. In some cases they are regular, and after.

almost periodical; in others they occur every day or In an early stage neuralgic affections of the urethra every week. In one case which I treated they came are, generally speaking, of little importance. They on as the patient was about to sit down to dinner, are removed by an easy, and, in most cases, efficacious and in another occurred daily at four o'clock. As a method of treatment, to a knowledge of which I was general rule, I would say that the accesses are more led by what I had observed in lithotrity. Thus when prolonged and frequent in proportion to the date of a patient, affected with stone, presents all the signs the disease. When they approach the quotidian type, of excessive irritation at the moment that we are the patient is usually attacked towards evening, as about to operate, one would imagine that the necesdigestion commences, or during the night. They are excited by want of occupation or distraction, by anything which increases the sensitiveness of the patient, and chiefly by the fear of not being able to make water freely. The less urine contained in the bladder, the greater the difficulty in passing it, and the more severe is the pain. We hardly ever observe any febrile symptoms even when the violence and obstinacy of the pain have occasioned general emaciation.

The course of urethral neuralgia is always progressive, but varies according to the complications which may already exist, or those which fail not to arise during the progress of the disease. Thus, laying aside the modifications which must ensue from the coexistence of stricture, disease of prostate, calculus, $\& c .$, if we regard the affection as purely neuralgic, it is ccrtain that the accesses become more violent, prolonged, and frequent, in proportion as the disease is of longer duration.

When this has continued for a length of time, the merely nervous symptoms are accompanied by others, dependant on catarrh of the bladder, or some organic disease of the prostate, bladder, \&c. The local disorder has now made considerable progress; the functions of the rectum are disturbed, and the general health suffers. Under these circumstances, it is extremely difficult to ascertain the existence of the primary neuralgia, unless the patient is able to give a very accurate history of the origin and progress of his disease. They generally confine themselves to vague reminiscences, and seldom call on the medical man before the symptoms have acquired a considerable degree of intensity.

Now, at this period the diagnosis is often very diff. cult, yet the question is much simplified when we ascertain that there is no stricture, disease of the prostate, or stone in the bladder.

The effect of examination of the genito-urinary organs is of great importance towards ascertaining the existence of urethral neuralgia, for in short cases they relieve, rather than exasperate, the disease. And here I would direct attention to a point which often gives rise to errors that belong rather to the person who practises than to the science. Many surgeons, not frequently in the habit of passing a bougie, find some difficulty in introducing this instrument beyond the curve of the urethra; the patient complains of cxcessive pain at this point, and the existence of stricture is rather hastily admitted.

There are, however, certain cases which baffle all our means of examination. In some patients the bladder or prostate is the seat of organic disease, accompanied by such excessive irritability of the urethra, that no instrument can be tolerated for a moment. It is also difficult to ascertain whether the organic diseases alluded to preceded or succeeded tion; but I have frequently seen the symptoms diminish, or even disappear, after the manipulations, and hence was led to think that neuralgic affections of the urethra and neck of the bladder might be advantageously treated by an attempt at modifying the sensibility of these parts. The event has fully justified my conclusion.

I shall now briefly explain the mode of treatment which I adopt in the different cases. When the diagnosis has been correctly formed, the indications of treatment are reduced to three-viz.,

1. To diminish, by the contact of a foreign body, the morbid sensibility of the urethra.

2. To excite a temporary disturbance of function, and thus break through the acquired habit in the part.

\section{To displace the irritation.}

In some cases a cure is obtained by fulfilling the first indication alone. Surgeons are now well aware that when a bougie has been allowed to remain for some time in the urethra, the canal becomes nearly insensible of its presence. I have applied this fact to the treatment of urethral neuralgia. The first step, then, in the treatment consists in passing into the urethra a soft, middle-sized bougie; this is allowed to remain in the canal for ten minutes, then withdrawn, and the operation repeated on the next and following days. The size of the bougie may be increased according to circumstances. Even in cases of long standing, from ten to fifteen applications of the bougie are sufficient; in more recent cases the cure is quicker. In all cases the symptoms are rapidly alleviated, and the use of the bougie should not be suspended until it passes the urethra without exciting any pain or uneasy sensation. It is necessary to employ, towards the end of the treatment, bougies of from two and a half to three lines in diameter, for we thus discover any incipient stricture, which might escape detection by a smaller instrument. Should this means fail, we have recourse to a more powerful mode of disturbing the sensibility of the part, by passing a catheter or a lithotritic instrument. I have seen a great many cases in which neuralgia of the urethra was cured by the introduction of the instrument, for the purpose of ascertaining the presence of a calculus. When the disease is more obstinate, and accompanied by atony of the bladder, much benefit will result from injecting pure water into the bladder, the temperature of the fluid being gradually reduced. Irrigations are a still more powerful means, and from them I have obtained the best results, even in cases which seemed desperate. Should the method now described fail, we have recourse to revulsions and counter-irritation. The latter is best applied to the hypogastric region or perineum. Cold douches are 
excellent ; if more powerful counter-irritation be required, the tartar emetic ointment must be applied over the hypogastrium, and repeated, if necessary, after the desiccation of the pustules. Regulation of the bowels is a matter of some importance, for the disease may be kept up by constipation. It is likewise necessary to mitigate the irritating quality of the urine, when the latter is high colored and scanty. Baths, emollient clysters, diluents, alkalies, mild regimen, are indicated in these cases.

Such are the means which experience has sanctioned as most efficacious in the treatment of urethral neuralgia. They sometimes fail, however, and, to relieve the patient's sufferings, more powerful means, as the use of caustics, forced injections, setons, \&c., have been recommended; I have myself found most benefit from slight but extensive applications of caustic; but the practitioner should always bear in mind that these powerful means will fail unless he take care to diminish previously the morbid sensibility of the urethra.-From M. Civiale on Diseases of the Urethra.

\section{$\overline{\mathrm{CASE}}$}

OF

WOUND IN THE THROAT, IN WHICH THE COMMON CAROTID ARTERY WAS TIED. By William Hey, Esq., Jun., Surgeon to the Leeds Infirmary, $x$.

A man, aged forty-five, whilst in a state of derangement, occasioned principally by excessive drinking, attempted to commit suicide by cutting his throat, on Wednesday, April 27. The wound, which was inflicted with a pen-knife, was not more than half an inch in length, but deep. It was situated on the left side of the neck, on a level with the upper part of the larynx, about midway between that and the anterior margin of the sterno-cleido-mastoideus muscle, and penetrated in a direction rather obliquely upwards. A surgeon was immediately sent for, but, before he arrived, the hæmorrhage had entirely ceased, and he therefore simply dressed the wound. A few days afterwards the wound and neighbouring parts were attacked with inflammation of an erysipelatous character, which, however, yielded to the remedies employed. Early on Saturday morning, May 7 (the eleventh day after the infliction of the wound), hamorrhage took place, in consequence of which Mr. Sharpe, the surgeon in attendance, requested my assistance. Before $I$ got to the patient the hæmorrhage had again ceased, and, as the quantity of blood lost had not been considerable, it was not thought necessary to do anything at that time. On Monday evening, May 9, the hæmorrhage returned. I then thought it necessary to secure the bleeding vessel, but the patient refused to submit to any operation. On the following evening it recurred for the third time, and the patient then, being convinced of the dangerous nature of the case, no longer refused his consent to what was judged necessary to be done. The plan upon which we proposed to proceed was this-tirst, to enlarge the wound, to turn out the coagulum, so as to expose the bleeding vessel, and, if possible, to tic it above and below the wound; if that should be found impracticable, then to continue the incision downwards, and tic the common carotid. On laying open the sac, and turning out the coagulum, such an impetuous gush of blood took place as plainly showed it would be impossible to accomplish the first object; and, therefore, the finger of an assistant being placed on the wound of the artery, I proceeded to tie the common carotid. The operation was attended with a good deal of difficulty, on account of the swelling, and still more of the change in the structure of the parts, consequent upon the previous inflammation. The cellular tissue was quite consolidated by the effusion of fibrine, so that I could make no impression upon it with the handle of the knife or the point of a director, but was obliged to work with the point of the knife in a deep cavity, and almost in the dark, the operation being performed by candle-light. The artery was, however, at length exposed, and tied immediately above the omo-hyoideus muscle. As soon as the ligature was tightened the hæmorrhage ceased. I then endeavoured to find the original wound of the artery, but, in consequence of its situation at the bottom of a deep cavity, and the diseased state of the parts, I did not succeed. For some days afterwards the patient had a considerable degree of fever; this seemed, however, to be subsiding, and I did not see or hear anything more of him until Sunday, June 22, the thirteenth day after the operation. Mr. Sharpe informed me, on the morning of that day, that while dressing the wound on the preceding day, a few drops of blood had ap. peared. An appointment was made for a meeting on the following morning, but the same afternoon I received an urgent message, requesting my immediate attendance, on account of a return of the bleed. ing, but before $I$ arrived at the patient's house he was dead. It appeared, however, on inquiry, that the quantity of blood lost had been small, and not sufficient to produce death, but that he had sunk from the effects of the irritative fever under which he had labored. The account given to me by the persons who were about him was, that he suddenly complained of a sense of suffocation, in consequence of which he made a violent effort to raise himself into the erect posture, and by that effort produced a flow of blood, but that he was, in fact, dying at the time. It was the last effort of life.

Upon examining the state of the parts after the death of the patient, the artery in the situation of the ligature was found in a perfectly satisfactory state, being firmly plugged with a coagulum nearly an inch in length. It was evident, therefore, that the bleeding had proceeded from the original wound.

This result $I$ rather anticipated, or at least feared, in consequence of the artery having been tied only below the wound; for whenever a considerable artery is wounded without being completely divided, I believe the only certain method of preventing secondary hæmorrhage is to apply a ligature both above and below the wound.

Cases may, however, occur in which this would be im. practicable, or attended with so much difficulty and uncertainty as to justify a reliance upon the simpler method of tying the artery between the wound and the heart, and the case $I$ have related was such an one. It was found, upon examination of the parts after death, that the original wound was in the external carotid just at the point when one of its principal branches (the facia 